ARTICLE

Received 23 Aug 2013 | Accepted 29 Oct 2013 | Published 27 Nov $2013 \quad$ DOl: 10.1038/ncomms3834

\title{
The TSC-mTOR pathway regulates macrophage polarization
}

Vanessa Byles ${ }^{1, \star}$, Anthony J. Covarrubias ${ }^{1, \star}$, Issam Ben-Sahra1, Dudley W. Lamming $2,3,4,5,6$, David M. Sabatini ${ }^{2,3,4,5,6}$, Brendan D. Manning ${ }^{1} \&$ Tiffany Horng ${ }^{1}$

Macrophages are able to polarize to proinflammatory M1 or alternative M2 states with distinct phenotypes and physiological functions. How metabolic status regulates macrophage polarization remains not well understood, and here we examine the role of mTOR (mechanistic target of rapamycin), a central metabolic pathway that couples nutrient sensing to regulation of metabolic processes. Using a mouse model in which myeloid lineage-specific deletion of $\operatorname{Tsc}\left(\mathrm{Tsc}^{\Delta / \Delta}\right.$ ) leads to constitutive mTOR complex 1 (mTORC1) activation, we find that $\mathrm{TsC}^{\mathrm{L} / \Delta}$ macrophages are refractory to $\mathrm{L}$-4-induced $M 2$ polarization, but produce increased inflammatory responses to proinflammatory stimuli. Moreover, mTORC1-mediated downregulation of Akt signalling critically contributes to defective polarization. These findings highlight a key role for the mTOR pathway in regulating macrophage polarization, and suggest how nutrient sensing and metabolic status could be 'hard-wired' to control of macrophage function, with broad implications for regulation of type 2 immunity, inflammation and allergy.

\footnotetext{
${ }^{1}$ Department of Genetics \& Complex Diseases, Harvard School of Public Health, Boston, Massachusetts 02115, USA. ${ }^{2}$ Whitehead Institute for Biomedical Research, Cambridge, Massachusetts 02142, USA. ${ }^{3}$ Department of Biology, MIT, Cambridge, Massachusetts 02139, USA. ${ }^{4}$ Howard Hughes Medical Institute, MIT, Cambridge, Massachusetts 02139, USA. ${ }^{5}$ Broad Institute of Harvard and MIT, Seven Cambridge Center, Cambridge, Massachusetts 02142 , USA. ${ }^{6}$ The David H. Koch Institute for Integrative Cancer Research at MIT, Cambridge, Massachusetts 02139, USA. * These authors contributed equally to this work. Correspondence and requests for materials should be addressed to T.H. (email: thorng@hsph.harvard.edu).
} 
$\mathrm{M}$ acrophages have a dynamic role in host defense and maintenance of tissue homoeostasis. This necessitates a delicate balance between their proinflammatory and immunomodulatory functions to ensure appropriate responses to environmental stimuli. Macrophages can be broadly classified into M1 (classical) and M2 (alternative) subtypes based on function. M1 macrophages are activated by LPS and/or IFN- $\gamma$ to elaborate proinflammatory cytokine production and tissue inflammation $^{1}$. Conversely, M2 macrophages are stimulated by Th2 cytokines IL-4 and/or IL-13 to promote helminthic immunity, fibrosis, allergy and immunomodulation ${ }^{2}$. Stimulation of macrophages with IL-4 and IL-13 leads to activation of the transcription factor STAT6, which is indispensable for M2 polarization ${ }^{3}$. In addition, activation of the nuclear receptors PPAR $\gamma$ and PPAR $\delta$ is necessary for full implementation of the M2 programme ${ }^{4,5}$. A hallmark of M2 macrophages is an increase in Arginase-1 gene expression and activity $^{2}$, which converts L-arginine to L-ornithine to promote polyamine synthesis and tissue repair ${ }^{6}$. The $\mathrm{M} 2$ programme is also characterized by upregulation of C-type lectins, mannose receptor, chitinase family proteins, resistin-like molecules and Interleukin-10, all of which contribute to immunomodulatory function ${ }^{7}$. Importantly, distinct metabolic programs are required to support energy demands of M1 and M2 macrophages. M1 macrophages rely primarily on glycolytic metabolism, mediated by HIF- $1 \alpha$, whereas M2 macrophages utilize fatty acid oxidation mediated by PPAR $\gamma$ and the transcriptional coactivator, PGC- $1 \beta$ (refs 3,8,9). This suggests that macrophage metabolism and inflammatory phenotype are integrally linked, and hint at additional regulatory control of macrophage polarization by metabolic pathways.

The mechanistic target of rapamycin (mTOR) is a key nutrient/ energy sensor that couples nutrient availability to regulation of downstream metabolic processes such as protein synthesis, glycolysis and de novo lipogenesis ${ }^{10,11}$. mTOR, a serine/ threonine kinase, exists in a rapamycin-sensitive complex called mTORC1 that is negatively regulated by the tuberous sclerosis complex comprising TSC1 and TSC2 (ref. 12). Genetic loss of either TSC1 or TSC2 leads to constitutive mTORC1 activation ${ }^{13}$. Importantly, recent studies demonstrate that mTOR controls multiple aspects of T-cell biology including quiescence, activation and differentiation ${ }^{14}$. However, little is known regarding the role of $\mathrm{mTOR}$ in regulating macrophage activation.

In the current study, we elucidate a role for mTOR in macrophage polarization. We demonstrate that $T s c 1^{\Delta / \Delta}$ macrophages have a marked defect in M2 polarization in response to IL-4, whereas the inflammatory response to LPS is enhanced. Aberrant polarization is due, at least in part, to mTORC1mediated attenuation of Akt activity, which renders $T s c 1^{\Delta / \Delta}$ macrophages resistant to the immunomodulatory effects of Akt downstream of IL-4 and LPS signalling. Finally, we show that IL4 and chitin administration in $T s c 1^{4 / \Delta}$ mice recapitulates the defective M2 polarization in vivo.

\section{Results}

Constitutive mTORC1 activity impairs M2 polarization. The mTOR pathway integrates a variety of inputs to regulate cell growth and to balance anabolic and catabolic processes ${ }^{15}$. Interestingly, stimulation of bone marrow-derived macrophages (BMDMs) with IL-4 or LPS resulted in mTORC1 activation as indicated by increased phosphorylation of the downstream targets S6K1 and 4E-BP1 (Fig. 1a), suggesting that the mTORC1 pathway may coordinate metabolic changes during macrophage activation. To examine this hypothesis, we utilized a model of myeloid-specific Tsc1 deficiency in which mTORC1 is constitutively active. Tscl loxP/loxP $L y s M C r e$ mice and Tscl loxP/ loxp controls are herein referred to as Tsc1 ${ }^{\Delta / \Delta}$ and Tsc1 ${ }^{f l f l}$, respectively. Immunoblotting confirmed that TSC1 is absent in the $T s c 1^{\Delta / \Delta}$ BMDMs (Fig. 1b). TSC2 protein level was also diminished (Fig. 1b), as TSC1 stabilizes TSC2 (ref 16). Tsc1 $1^{\Delta / \Delta}$ BMDMs displayed constitutive phosphorylation of the mTORC1 downstream targets S6K1 and 4E-BP1, as well as the S6K1 target ribosomal S6, all of which were sensitive to the mTORC1-specific inhibitor, rapamycin (Fig. 1b). Furthermore, $T s c 1^{\Delta / \Delta}$ BMDMs seemed to differentiate normally in vitro and expressed similar levels of the macrophage markers F4/80 and CD11b (Supplementary Fig. S1). As reported in other models of TSC deficiency ${ }^{17}, T s c 1^{0 / 4}$ BMDMs were larger (Supplementary Fig. S1) due likely to a role of mTORC1 in regulating cell size $^{18}$. These observations confirmed constitutive mTORC1 activation in $T s c 1^{\Delta / \Delta}$ BMDMs and established the validity of our genetic model.

To assess macrophage polarization in $T s c 1^{\Delta / \Delta}$ BMDMs, we used LPS treatment to promote an M1-like phenotype and IL-4 stimulation to induce an M2 phenotype. We found that LPStreated $T s c 1^{\Delta / \Delta}$ BMDMs secreted more of the proinflammatory cytokines IL- 6 and TNF- $\alpha$, but less of the anti-inflammatory cytokine IL-10 (Fig. 1c). Given the enhanced responses of $T s c 1^{\Delta / \Delta}$ BMDMs to LPS, we postulated that M2 polarization could be defective. Indeed, $T s c 1^{\Delta / \Delta}$ BMDMs failed to fully upregulate the M2 programme, with significant reductions in Arg1, Mgll, Mgl2, Ym1, Fizz1 and Pgc1- $\beta$ expression (Fig. 1d). Arginase-1 activity assessed by urea production was reduced, correlating with lower levels of Arg1 messenger RNA (Fig. 1e). IL-4-stimulated fatty acid oxidation, another hallmark feature of M2 macrophages, was also defective (Fig. 1f). This finding is consistent with the threefold reduction in Pgc1- $\beta$ (Fig. 1d), a known mediator of fatty acid oxidation in M2 macrophages ${ }^{9}$. Interestingly, M2c polarization triggered by IL-10 stimulation was also deficient in $T s c 1^{\Delta / \Delta}$ BMDMs (Supplementary Fig. S2a), which may indicate impaired orchestration of anti-inflammatory responses during tissue remodelling or wound healing ${ }^{19}$. Macrophage activation to the M2b phenotype (by treatment with LPS/immune complexes), which has features of both M1 and M2 macrophages ${ }^{20,21}$, was not affected (Supplementary Fig. S2b). Taken together, our findings demonstrate that aberrant mTORC1 activation critically modulates macrophage polarization. Impaired induction of $P g c-1 \beta$ and fatty acid oxidation also highlights a key role of mTOR in orchestrating macrophage cellular metabolism.

STAT6 and PPAR $\gamma$ activity are normal in $T s c 1^{4 / \Delta}$ BMDMs. IL$4 \mathrm{R}$ signalling leads to activation of JAK1/JAK3 and tyrosine phosphorylation of the transcription factor STAT6, enabling nuclear translocation ${ }^{2}$ and induction of target genes such as Ppary and Arg1 (ref. 3). To interrogate the mechanism underpinning defective M2 polarization in $T s c 1^{\Delta / \Delta}$ BMDMs, we first examined signal transduction downstream of the IL-4R. $T s c 1^{\Delta / \Delta}$ BMDMs expressed comparable levels of Jak1, Jak3 and $I L-4 r \alpha$ messenger RNA as well as STAT6 protein at basal state (Fig. 2a, Supplementary Fig. S3a). Following IL-4 stimulation, STAT6 was tyrosine phosphorylated consistent with normal activation (Fig. 2a). Chromatin immunoprecipitation assays indicated comparable IL-4-induced recruitment of STAT6 to the promoter of the Arg1 gene (Supplementary Fig. S3b). Furthermore, IL-4-inducible STAT6 transcriptional activity as measured by a STAT6 reporter assay was unaffected (Fig. 2b). Finally, normal induction of some M2 genes in $T s c 1^{\Delta / \Delta}$ BMDMs, including the STAT6-dependent gene Ppary ${ }^{22}$ (Fig. 2c), indicates no general defect in the expression or activation of STAT6, the master regulator of M2 gene induction. IL-4 signalling can 
a

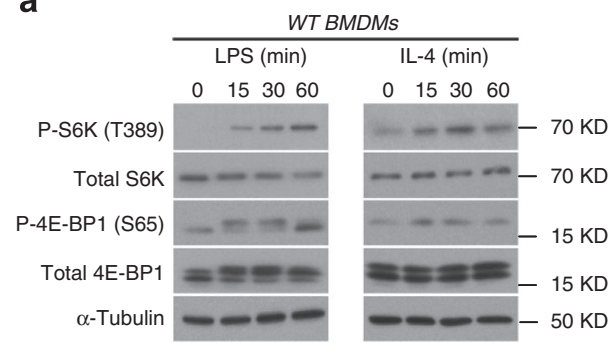

C
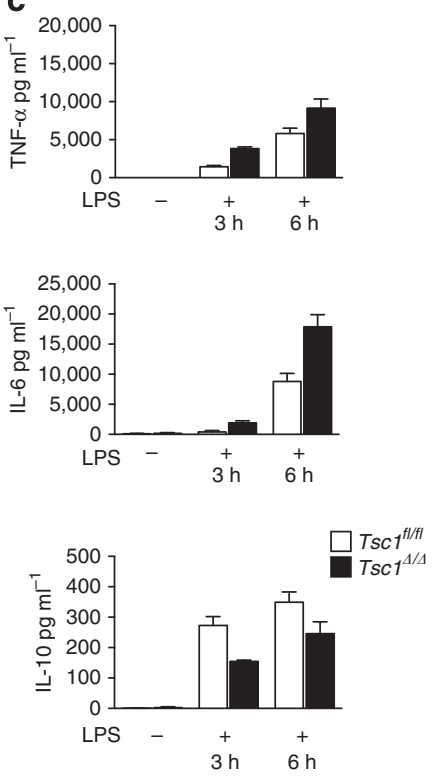

b

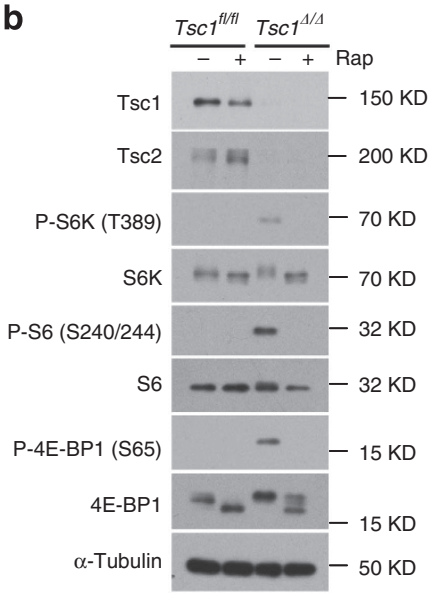

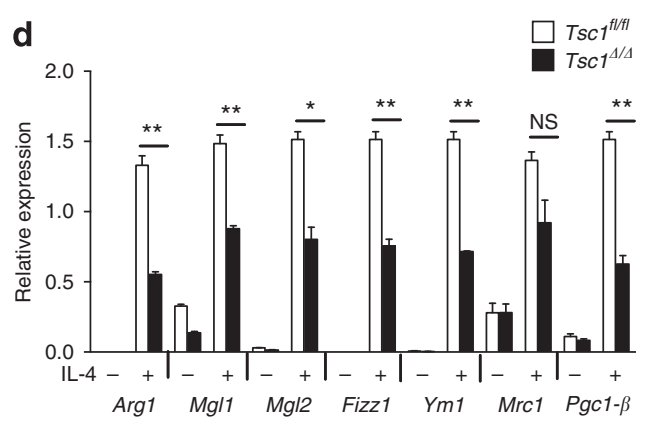

e

$\mathbf{f}$
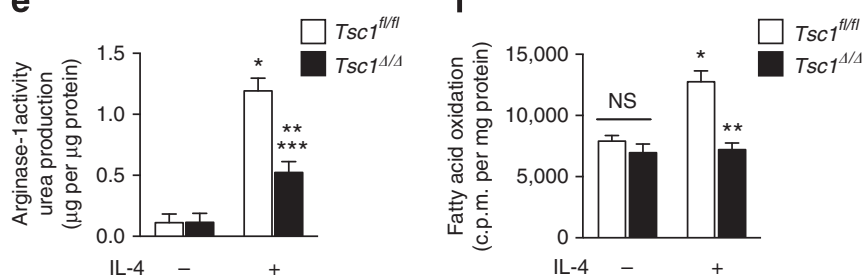

Figure 1 | Tsc1 ${ }^{4 / \Delta}$ BMDMs have defective M2 polarization and enhanced responses to LPS stimulation. (a) Immunoblot analysis of WT BMDMs stimulated with LPS or IL-4 for 15-60 min as indicated. (b) Immunoblot analysis of lysates from Tsc $T^{f l / f l}$ and $T s c 7^{4 / \Delta}$ BMDMs treated with or without rapamycin for $15 \mathrm{~h}$. (c) Measurement of TNF- $\alpha$, IL- 6 and IL-10 secretion by ELISA after treatment with LPS for $3 \mathrm{~h}$ and $6 \mathrm{~h}$, $(n=2$ representative experiments). (d) Expression of M2 genes in $T s c f^{f / / f l}$ and $T s c 7^{4 / \Delta}$ BMDMs after treatment with IL-4 for $24 \mathrm{~h}(n=3) .{ }^{\star} P<0.05,{ }^{\star \star} P<0.01,{ }^{\star \star \star} P P<0.001$. (e) Urea production normalized to total protein in $T s c f^{f l / f I}$ and $T s c 7^{4 / \Delta}$ BMDMs stimulated as in $(\mathbf{c}),(n=4) * P<0.001$ for untreated versus $I L-4$ for

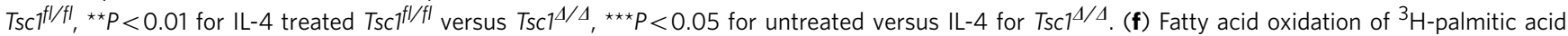
presented as counts per minute normalized to milligram of total protein after $36 \mathrm{~h}$ treatment with IL-4, $(n=3)$. ${ }^{\star} P<0.01$ for untreated versus IL-4 in $T s c T^{f l / f l},{ }^{\star \star} P<0.01$ for $\mathrm{IL}-4$ treated $T s c T^{f l / f l}$ versus $T s c 1^{4 / \Delta}$. Graphs are shown as mean \pm s.e.m. $P$ values were determined using Student's $t$-tests.

activate STAT1 in some contexts ${ }^{23,24}$, but not in macrophages (Supplementary Fig. S3c) consistent with a prior report, excluding a role for impaired STAT1 activation in the phenotype of Tsc1 $1^{\Delta / 4}$ $\mathrm{BMDMs}^{25}$.

Because previous studies indicate a key role for the nuclear receptors PPAR $\gamma$ and PPAR $\delta$ in M2 polarization, we next turned to an analysis of their expression and activity in $T s c 1^{\Delta / \Delta} \mathrm{BMDMs}$. First, we showed comparable expression of PPAR $\gamma$ and PPAR $\delta$ in Tsc1 $1^{f l f l}$ and $T s c 1^{\Delta / \Delta}$ BMDMs, at basal state and after IL-4 stimulation (Fig. 2c). As the expression does not necessarily reflect functional activity, we examined PPAR $\gamma$ activity in $T s c 1^{\Delta / \Delta}$ BMDMs. We found that IL-4-induced comparable expression of the canonical PPAR $\gamma$-dependent genes Fabp4 (refs 22,26) and Cd36 (ref. 27) (Fig. 2d). Furthermore, the PPAR $\gamma$ agonist troglitazone synergized with IL-4 to a similar extent in Tsc1 ${ }^{f l f l}$ and $T s c 1^{\Delta / \Delta}$ BMDMs (Fig. 2d). Analogous findings were obtained with the PPAR $\delta$-dependent gene $\operatorname{Atgl}^{28}$ using the PPAR $\delta$ agonist GW501516 (Supplementary Fig. S4). To corroborate gene 
a

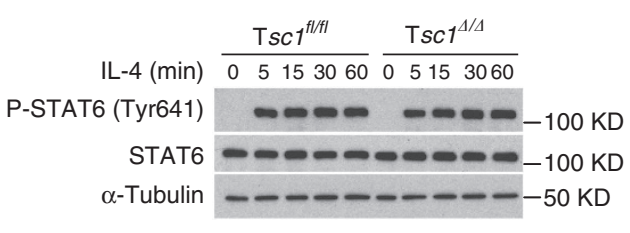

b STAT6 reporter assay

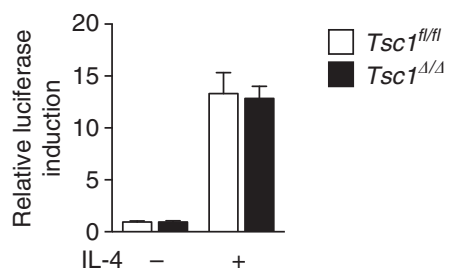

C
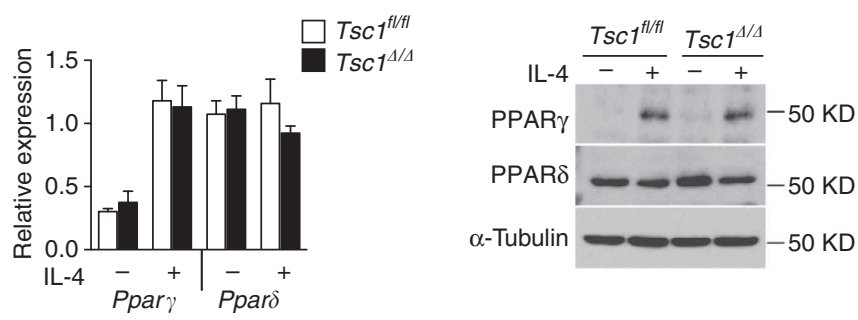

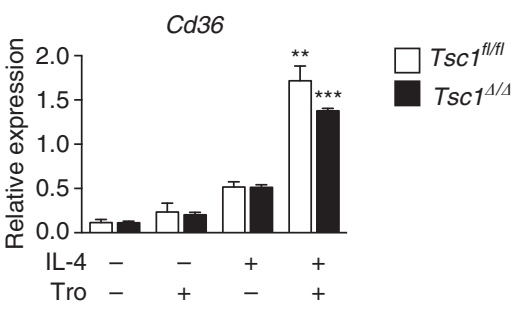

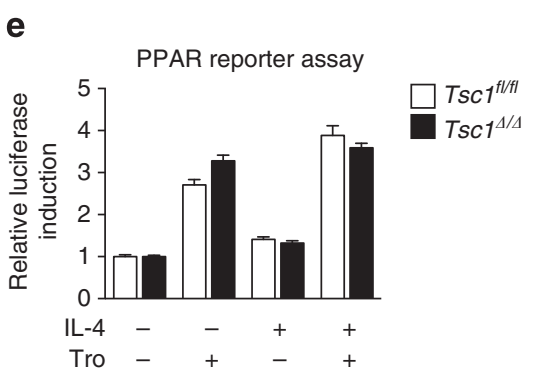

Figure 2 | STAT6 and PPAR $\gamma$ activity are normal in Tsc1 ${ }^{\Delta / \Delta}$ BMDMs. (a) Immunoblot analysis of lysates from $T s c 7^{f l / f l}$ and $T s c 7^{\Delta / \Delta}$ BMDMs stimulated with IL-4 for 5-60 min. (b) STAT6 luciferase reporter assay in Tsc $\mathrm{fl}^{\mathrm{flfl}}$ and $\mathrm{Tsc}^{1 / \Delta}$ BMDMs. Data shown as fold induction of firefly luciferase activity normalized to renilla luciferase for IL- 4 treatment relative to untreated sample ( $n=2$ experiments performed in duplicate). (c) Gene expression and immunoblots for PPAR $\gamma$ and PPAR $\delta$ in $T s c f^{f / f l}$ and $T s c 7^{4 / \Delta}$ BMDMs after treatment with IL-4 for $24 \mathrm{~h}$. Gene expression data are shown as mean \pm s.e.m. $(n=3)$. (d) Expression of PPAR $\gamma$-dependent genes in $T s c f^{f / f l}$ and $T s c 1^{4 / \Delta}$ BMDMs treated with IL-4 in the presence or absence of troglitazone for $24 \mathrm{~h}$. DMSO vehicle was used as control, $(n=3)$. ${ }^{\star} P<0.05$ for IL-4 treated Tsc $f^{f / f f l}$ and $T s c 1^{4 / 4},{ }^{\star \star} P<0.01,{ }^{\star \star \star} P<0.001$ for IL-4 versus IL-4 + Tro. (e) PPAR luciferase reporter assay in $T s c f^{f l / f l}$ and $T s c 7^{\Delta / \Delta}$ BMDMs. Data shown as fold induction of firefly luciferase activity normalized to renilla luciferase for IL-4 or troglitazone treatment relative to untreated sample (representative of three experiments performed in triplicate). Graphs are shown as mean \pm s.e.m. $P$ values determined using Student's $t$-tests.

expression data, we used a PPAR reporter assay to assess PPAR transcriptional activity, and found commensurate induction in $T s c 1^{f l / f l}$ and $T s c 1^{\Delta / \Delta}$ BMDMs (Fig. 2e). Taken together, these findings indicate a selective defect in $\mathrm{M} 2$ polarization in $T s c 1^{\Delta / \Delta}$ BMDMs that may not be due to defects in STAT6, PPAR $\gamma$, or PPAR $\delta$ expression or activity.

mTORC1 activity attenuates IL-4-induced Akt activation. The data above suggest that $T s c 1^{\Delta / \Delta}$ BMDMs may not be able to activate some signals downstream of IL-4R. We turned our attention to IRS2/PI3K/Akt signalling, as this pathway is engaged by the IL-4R in parallel to the JAK/STAT6 pathway ${ }^{29,30}$. PI3K activation leads to increased activity of mTORC2, which phosphorylates Akt at S473 to activate the protein and promote membrane localization. In addition to S473, Akt is critically controlled by phosphorylation at T308, a step mediated by PDK1 (Fig. 3a). Thus we examined S473 and T308 phosphorylation as readouts of Akt activity, as well as phosphorylation of the downstream Akt targets FOXO1, PRAS40 and GSK-3 (ref. 31) (Fig. 3a).

Interestingly, IL-4-stimulated $T s c 1^{\Delta / \Delta}$ BMDMs displayed a striking attenuation in Akt signalling as indicated by reduced phosphorylation of $\mathrm{Akt}^{\mathrm{S} 473}$ and $\mathrm{Akt}^{\mathrm{T} 308}$ (Fig. 3b). Consistently, phosphorylation of the Akt targets FOXO1 and PRAS40 was diminished (Supplementary Fig. S5a). GSK-3 phosphorylation was not reduced (Supplementary Fig. S5a), perhaps because of its regulation by multiple inputs ${ }^{32-35}$. Importantly, diminished Akt signalling has been noted in TSC-deficient cells during stimulation with insulin and other growth factors ${ }^{36-38}$. Such reduction of Akt activity is due to mTORC1-mediated negative feedback that impinges on multiple targets, including but not 
a

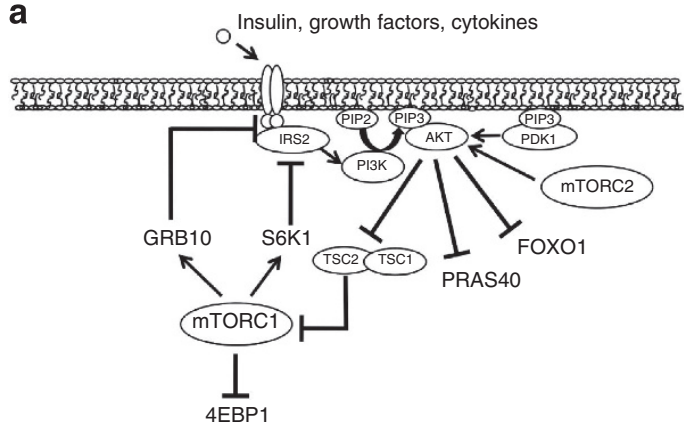

b

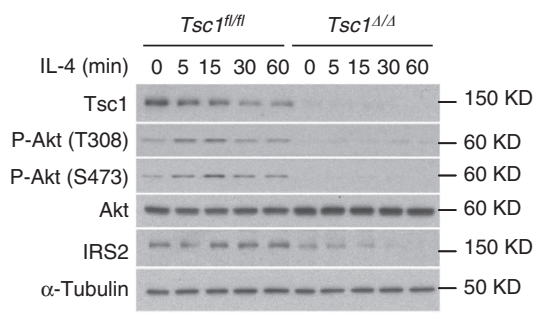

C

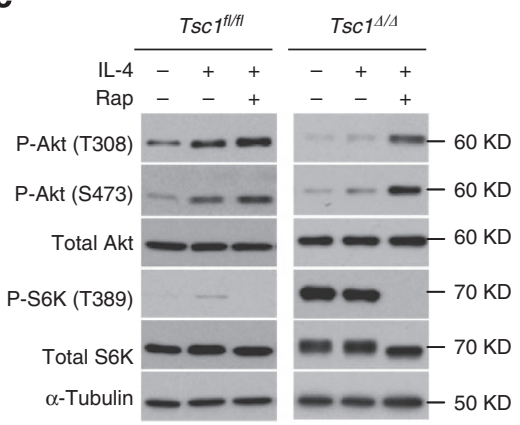

d
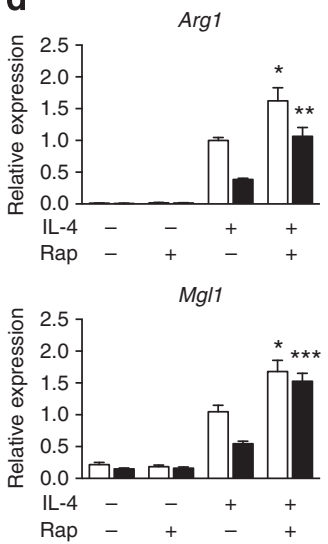

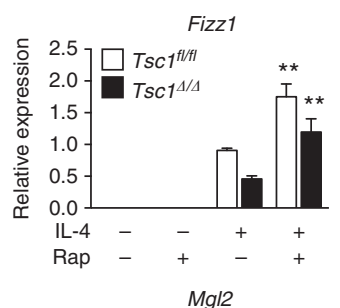

Mgl2

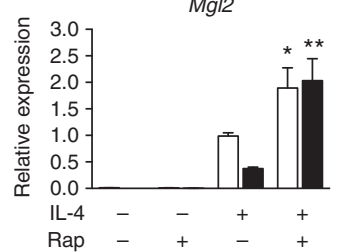

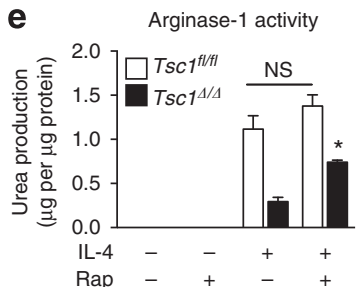

Figure 3 | Constitutive mTORC1 activity attenuates IL-4-induced Akt activation. (a) Overview of mTORC1 signalling downstream of IL-4, insulin and growth factors. Receptor activation engages the IRS/PI3K/Akt pathway. PI3K converts PIP2 to PIP3 thus recruiting PDK1 and Akt to the plasma membrane, enabling PDK1-mediated phosphorylation of Akt on T308. PI3K also activates mTORC2, which phosphorylates Akt on S473. Thus activated, Akt can phosphorylate downstream targets to regulate their activity. One consequence of Akt activation is increased mTORC1 activity, which feeds back to attenuate IRS2/PI3K/Akt signalling through multiple mechanisms, including reducing levels of IRS2 while increasing levels of GRB10. (b) Immunoblot analysis of lysates from $T s c T^{f l / f l}$ and $T s c T^{4 / \Delta}$ BMDMs stimulated with IL-4 for 5-60 min. (c) Immunoblot analysis of lysates from $T s c f^{f l / f l}$ and $T s c 7^{4 / \Delta}$ BMDMs stimulated with IL-4 for $20 \mathrm{~min}$ in the presence or absence of rapamycin ( $20 \mathrm{nM}, 1 \mathrm{~h}$ pretreatment). DMSO vehicle was used as control. (d) Expression of $\mathrm{M} 2$ genes in $T s c T^{\mathrm{fl} / \mathrm{fl}}$ and $T s c T^{\Delta / \Delta}$ BMDMs after treatment with $\mathrm{IL}-4$ for $15 \mathrm{~h}$ in the presence or absence of rapamycin (20 $\mathrm{nM}$, $1 \mathrm{~h}$ pretreatment). $(n=5) .{ }^{\star} P<0.05,{ }^{\star \star} P<0.01,{ }^{\star \star \star} P<0.001$ for IL-4 versus IL-4 + rap. (e) Urea production normalized to total protein in Tsc $\mathrm{T}^{f l / f l}$ and $T s c 7^{4 / 4}$ BMDMs stimulated with IL-4 for $20 \mathrm{~h}$ in the presence or absence of rapamycin ( $20 \mathrm{nM}, 1 \mathrm{~h}$ pretreatment). $(n=5)$, ${ }^{\star} P<0.001$. Graphs are shown as mean \pm s.e.m. $P$ values were determined using Student's $t$-tests.

limited to IRS1/2 degradation ${ }^{39,40}$ and phosphorylation and stabilization of GRB10 (refs 41,42) (Fig. 3a). Although such mTORC1-mediated negative feedback is well defined for insulin signalling, little is known regarding its role in the regulation of cytokine signalling. Interestingly, we found that $T s c 1^{\Delta / \Delta}$ BMDMs display reduced IRS2 levels in response to IL-4 treatment (Fig. 3b). This is likely to contribute to mTORC1-mediated attenuation of Akt signalling, as IRS2 has been implicated in Akt activation during IL-4 stimulation ${ }^{29}$. Increased levels of phosphorylated and total GRB10 (Supplementary Fig. S5b) may also have a role given that GRB10 inhibits signalling downstream of RTKs ${ }^{43-45}$. Thus increased mTORC1 activity in our model is likely to attenuate Akt signalling at least in part through IRS2 and GRB10. PDK1, the Akt T308 kinase, has constitutive kinase activity and is critically regulated by PIP3-mediated recruitment to the plasma membrane ${ }^{46}$, thus normal PDK1 activity in $T s c 1^{4 / \Delta}$ BMDMs (Supplementary Fig. S5c) also supports our model that attenuated Akt signalling in $T s c 1^{4 / \Delta}$ BMDMs occurs at a receptor proximal step upstream of PI3K-mediated PIP3 production (Fig. 3a). Finally, LPS-mediated Akt activation was diminished in $T s c 1^{\Delta / \Delta}$ BMDMs, as evidenced by a defect in phosphorylation of Akt and the Akt target FOXO1 (Supplementary Fig. S5d). Collectively, these findings demonstrate that mTORC1-mediated negative feedback mechanisms converge to ultimately attenuate Akt signalling in $T s c 1^{\Delta / \Delta}$ BMDMs.

Having shown a defect in Akt activation in $T s c 1^{\Delta / \Delta}$ BMDMs, we addressed a potential role in impaired M2 polarization. Previous studies have used rapamycin treatment to alleviate mTORC1-mediated negative feedback of Akt signalling and to interrogate the role of attenuated Akt activation in TSC-deficient 
models ${ }^{36-38}$. Accordingly, we found that rapamycin treatment of $T s c 1^{\Delta / \Delta}$ BMDMs rescued IL-4-inducible Akt signalling (Fig. 3c). Importantly, such treatment restored induction of M2 genes Arg1, Fizz1 and Mgl1/2 as well as Arginase activity (Fig. 3d,e). Together this suggests that deficient M2 polarization in $T s c 1^{\Delta / \Delta}$ BMDMs may be due to mTORC1-mediated negative feedback of Akt signalling. We note that rapamycin treatment of control $T s c 1^{f l / f l}$ BMDMs also modestly increased Akt signalling (Fig. 3c) as well as M2 responses (Fig. 3d,e), indicating that acute, signaldependent activation of mTORC1 during IL-4 signalling can also feedback to inhibit Akt activation.

Attenuated Akt signalling underlies aberrant polarization. Our findings linking reduced Akt activation to impaired M2 polarization in $T s c 1^{4 / \Delta}$ BMDMs (Fig. 3b-e) are interesting given that little is known regarding the role of Akt in this process. To address this directly, we treated WT BMDMs with MK-2206, an allosteric inhibitor of Akt. This led to a decrease in IL-4-inducible phosphorylation of $\mathrm{Akt}^{\mathrm{T} 308}$ and $\mathrm{Akt}^{\mathrm{S473}}$ as well as the Akt target FOXO1, but did not affect STAT6 phosphorylation (Fig. 4a). Importantly, pretreatment with MK-2206 reduced IL-4-mediated induction of Arg1, Fizz1, Mgl2 and Mgl1 (Fig. 4b), as well as Arginase-1 activity (Fig. 4c). Similar effects were observed with the structurally distinct Akt inhibitor Akt VIII (Supplementary Fig. S6a,b), indicating the specificity of the inhibitors. These findings argue that Akt may have an important role in M2 polarization. Together with the data in Fig. 3, they also support the idea that attenuated Akt signalling underpins defective M2 polarization in $T s c 1^{\Delta / \Delta}$ BMDMs.

Next, we took a genetic approach to rescue Akt signalling in $T s c 1^{\Delta / \Delta}$ BMDMs. As expected, retroviral transduction of $T s c 1^{\Delta / \Delta}$ BMDMs with myristylated-Aktl (myr-Akt) led to constitutive Akt signalling as indicated by high basal state $\mathrm{P}-\mathrm{Akt}^{\mathrm{T} 308}$ and P-Akt ${ }^{\$ 73}$ (Fig. 4d). Importantly, this was associated with increased induction of Arg1 and $M g l 1$ (Fig. 4e), as well as Arginase-1 activity (Fig. 4f), following IL-4 stimulation. Although ectopic expression of myr-Akt was insufficient to rescue Fizz1 and $M g l 2$ expression, this could be achieved in the context of rapamycin co-treatment (Supplementary Fig. S6c). Thus, myrAkt may not fully recapitulate IL-4-inducible Akt activation; or alternatively, rescue of Akt signalling is not sufficient for restoring Mgl2 and Fizzl expression, and some other consequence of constitutive mTORC1 activation is critically relieved by rapamycin treatment. Finally, to extend these studies to the proinflammatory responses, we examined myr-Akt expressing $T s c 1^{\Delta / \Delta}$ BMDMs following LPS treatment. We observed significantly reduced expression of $I l-6$ and Tnf $\alpha$ but increased expression of Il-10 (Fig. 4g and Supplementary Fig. S6d).

Deficient M2 polarization in $T s c 1^{\Delta / \Delta}$ mice. Finally, we asked whether TSC1 deficiency would impair M2 polarization in vivo. We used intraperitoneal (IP) injection of an IL-4/IL-4 antibody complex to elicit IL-4-dependent recruitment and in situ proliferation of $\mathrm{M} 2$ macrophages ${ }^{47}$ in $T s c 1^{f l f l}$ and $T s c 1^{4 / \Delta}$ mice. Strikingly, induction of most M2 genes was decreased in peritoneal exudate cells (PECs) from Tsc1 $1^{\Delta / \Delta}$ mice (Fig. 5a). To corroborate these findings, we used a model of chitin administration that triggers IL-4-dependent recruitment and polarization of M2 macrophages ${ }^{48,49}$. We observed a near universal reduction of $\mathrm{M} 2$ gene expression in $T s c 1^{\Delta / \Delta} \mathrm{PECs}$ (Fig. 5b), similar to the IL-4 injection. Collectively, the findings support our model that constitutive mTORC1 activity can attenuate macrophage M2 polarization in vivo.

\section{Discussion}

In this study, we use a novel model of myeloid-specific Tsc1 deletion and constitutive mTORC1 activity to elucidate mTORC1 function in macrophages. We found that $T s c 1^{\Delta / \Delta}$ BMDMs have enhanced proinflammatory cytokine production while IL-10 secretion is reduced, in line with a recent analysis of $T s c 1^{\Delta / \Delta}$ $\mathrm{BMDMs}^{17}$. Other studies reached conflicting conclusions, using small hairpin RNA knockdown in monocytes and dendritic cells ${ }^{50,51}$. Extending the analysis of mTORC1 function in macrophages, we showed that $T s c 1^{\Delta / \Delta}$ BMDMs are impaired in a

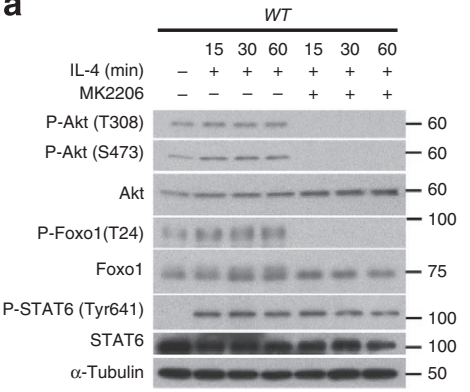

b

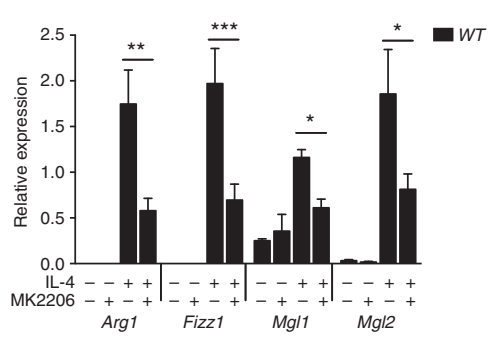

C

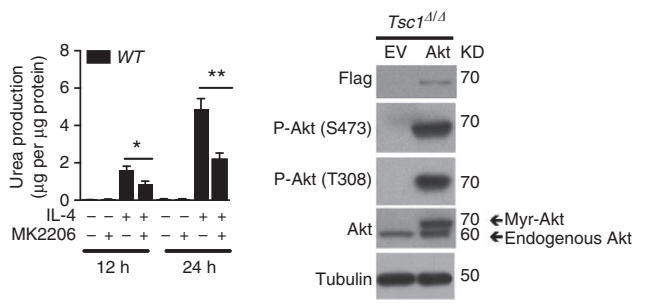

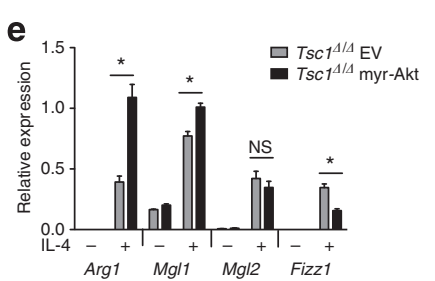
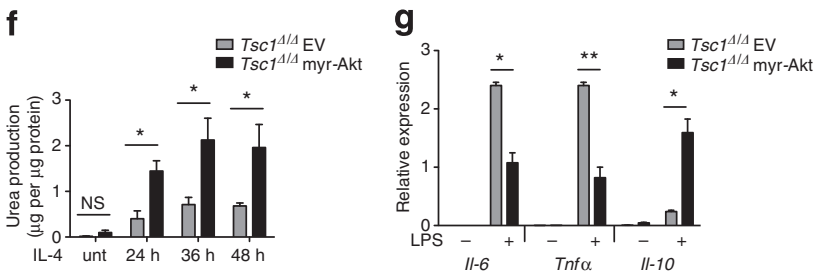

Figure 4 | Akt signalling is critical for polarization in $\mathbf{T s c 1}^{\mathbf{4} / \boldsymbol{A}}$ BMDMs. (a) Immunoblot analysis of WT BMDMs pretreated with MK-2206 for $1 \mathrm{~h}$ and treated with IL-4 for the indicated time points. $(\mathbf{b}, \mathbf{c})$ WT BMDMs were pretreated with MK-2206 or DMSO for $1 \mathrm{~h}$ before stimulation with IL-4 for $24 \mathrm{~h}$ and examination of $(\mathbf{b}) \mathrm{M} 2$ gene expression $(n=3)$ or $(\mathbf{c})$ urea production $(n=4)$. (d) Immunoblot analysis of $T s c 7^{4 / \Delta}$ BMDMs transduced with myr-flag-Akt or EV. (e,f) Myr-Akt Tsc7 ${ }^{4 / \Delta}$ BMDMs and EV Tsc7 ${ }^{\Delta / \Delta}$ BMDMs were stimulated with IL-4 followed $24 \mathrm{~h}$ later by analysis of (e) M2 gene

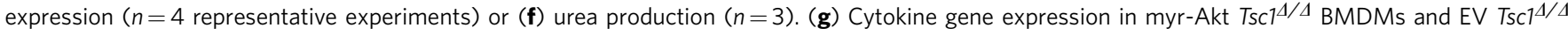
BMDMs stimulated with LPS for $6 \mathrm{~h}(n=3)$. Graphs are shown as mean \pm s.e.m., ${ }^{\star} P<0.05,{ }^{\star \star} P<0.01,{ }^{\star \star \star} P<0.001$. $P$ values were determined using Student's t-tests. 

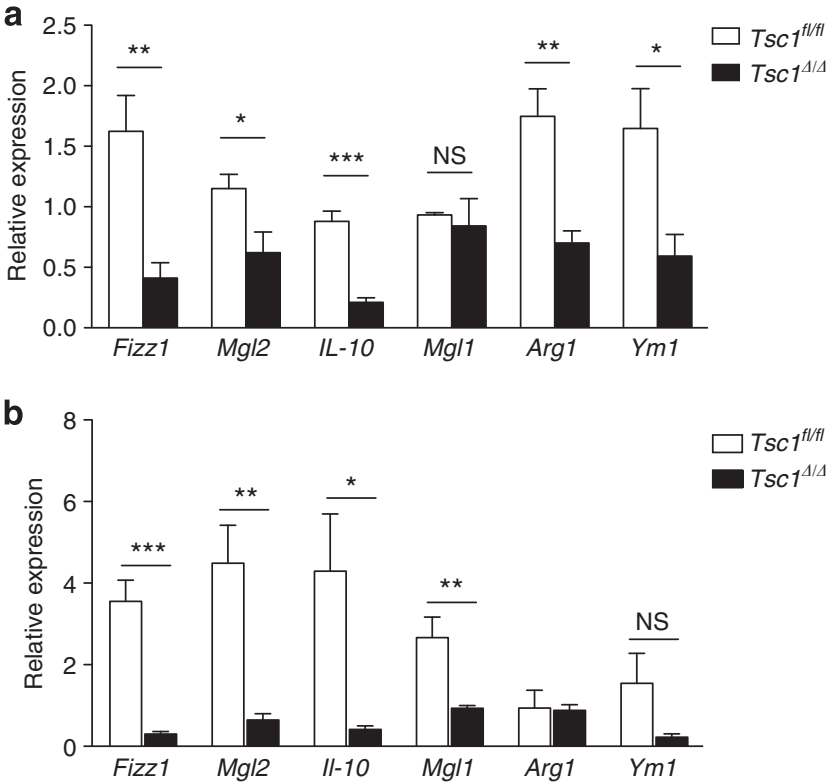

Figure 5 | M2 polarization in $\operatorname{Tsc1}^{\Delta / \Delta}$ mice is impaired in vivo. (a) M2 gene expression in PECs from $\mathrm{Tsc}^{\Delta / \Delta}$ and $T s c f^{\mathrm{fl} / \mathrm{fl}}$ mice 4 days post IP injection with $\mathrm{IL}-4 \mathrm{c}$ on days 0 and 2 ( $n=4$ mice per genotype). (b) M2 gene expression in PECs from male $T_{s c} 7^{4 / \Delta}$ and $T s c 1^{f l / f l}$ mice $48 \mathrm{~h}$ post IP injection with chitin ( $n=5$ mice per genotype). Data shown as mean \pm s.e.m., ${ }^{\star} P<0.05,{ }^{\star \star} P<0.01,{ }^{\star \star \star} P<0.001$. $P$ values were determined using Student's t-tests.

M2 polarization, expressing reduced levels of key M2 markers such as Arg1, Fizz1, Mgl1, Mgl2, Ym1 and Pgc-1 $\beta$ (Fig. 1d). Interestingly, the defect in M2 activation seems to be selective, as induction of PPAR $\gamma$ - and some PPAR $\gamma$-dependent genes (for example, Cd36 and Fabp4) occur normally (Fig. 2c,d). In addition, we find that $T s c 1^{\Delta / \Delta}$ BMDMs have diminished levels of Arginase- 1 activity and fatty acid oxidation, hallmark features of M2 macrophages (Fig. 1e,f). This suggests that mTORC1 may couple regulation of fatty acid oxidation to control macrophage polarization, consistent with the emerging view that macrophage cellular metabolism is closely linked to activation status $3,8,52$. Finally, we use models of IL- 4 and chitin injection to show that constitutive mTORC1 activity in myeloid lineage cells results in broad defects in M2 polarization in vivo (Fig. 5).

Normal activation of known regulators of M2 polarization, including STAT6, PPAR $\gamma$ and PPAR $\delta$ (Fig. 2), suggests that impaired M2 polarization in $T s c 1^{\Delta / 4}$ BMDMs may be due to block of a parallel signalling pathway. In addition to the JAK/ STAT6 pathway, IL-4R signalling engages IRS2/PI3K signalling in parallel to mediate Akt activation ${ }^{29,30}$. Although STAT6 is indispensable for M2 polarization $^{1,53}$, the role of Akt signalling has not been well characterized. Our findings suggest a critical role for mTORC1-mediated feedback inhibition of Akt signalling in $T s c 1^{\Delta / \Delta}$ BMDMs. In support of this, $T s c 1^{\Delta / \Delta}$ BMDMs display decreased IL-4-inducible Akt activation, as indicated by diminished $\mathrm{P}-\mathrm{Akt}^{\mathrm{T} 308}$ and P-Akt ${ }^{\mathrm{S473}}$ (Fig. 3b) and phosphorylation of the Akt targets FOXO1 and PRAS40 (Supplementary Fig. S5a). Importantly, rapamycin treatment (Fig. 3c-e) and ectopic expression of myr-Akt (Fig. 4d-f, Supplementary Fig. S6c) restore Akt activation simultaneous with rescue of M2 gene expression and Arginase activity. We suggest that decreased IRS2 (Fig. 3b) but increased GRB10 (Supplementary Fig. S5b) levels may contribute to mTORC1-mediated attenuation of Akt signalling, as IRS2 has been implicated in IL-4R signalling 54 , while GRB10 downregulates signalling downstream of $\mathrm{RTKs}^{43}$. Although other negative feedback mechanisms have been described in insulin signalling ${ }^{40,55}$ and may exist in our setting, they act synergistically and ultimately converge to attenuate Akt activation. Finally, we believe that feedback inhibition to Akt may also underlie the enhanced responsiveness of $T s c 1^{4 / \Delta}$ BMDMs to LPS stimulation. Although we have not extensively characterized the underlying mechanism(s) in this context, Akt activation in $T s c 1^{\Delta / \Delta}$ BMDMs is diminished following LPS signalling (Supplementary Fig. S5d), and its rescue with myr-Akt expression reduces the exaggerated responses (Fig. 4g).

Importantly, control of M2 polarization by Akt signalling is likely to extend beyond our genetic model to other settings, as pharmacological inhibition of Akt impairs M2 activation in wildtype BMDMs (Fig. 4b,c, Supplementary Fig. S6a,b). Moreover, rapamycin treatment of control BMDMs modestly increases Akt signalling and M2 responses (Fig. $3 \mathrm{C}-\mathrm{e}$ ). Thus our findings reveal a largely unappreciated role for Akt in synergizing with the STAT6 pathway to regulate full M2 polarization (Fig. 6a). Whether Akt promotes or inhibits inflammation downstream of LPS signalling is not entirely clear ${ }^{56}$, but at least in $T s c 1^{4 / \Delta}$ BMDMs, simultaneous mTORC1 activation and Akt attenuation lead to enhanced proinflammatory responses that can be rescued by restoring Akt signalling (Fig. $4 \mathrm{~g}$ ). The relevant Akt targets that regulate macrophage polarization are not well defined, but could include FOXO1 and CEBP $\beta^{57,58}$. Interestingly, macrophages deficient in Rictor, a subunit of the mTORC2 complex that phosphorylates Akt on S473, were deficient in some but not all Akt-dependent activities (Supplementary Fig. S7a) consistent with previous models of mTORC2 deficiency ${ }^{59,60}$ but polarized normally to the M2 phenotype (Supplementary Fig. S7b,c), and could serve as a plausible model to identify the relevant Akt targets controlling M2 activation.

Our studies indicate the existence of a mTORC1-Akt regulatory loop in the IL-4 signalling pathway that parallels that of the insulin pathway. In the latter, a feedback loop between mTORC1 and Akt-in which receptor engagement of the IRS/PI3K/Akt pathway leads to mTORC1 activation that feeds back to attenuate Akt signalling - is critical for transient, signal-dependent activation of these two signalling modules (Fig. 3a). Similarly, activation of the IRS2/PI3K/Akt pathway by IL-4 mediates mTORC1 activation (Fig. 1a, Supplementary Fig. S8) and as shown here, results in feedback inhibition of Akt signalling (Fig. 3a-c, Fig. 6a). Importantly, mTORC1 activity is critically modulated by nutrient/energy availability ${ }^{61}$, thus we propose that integration of the mTORC1-Akt regulatory loop into the IL-4 signalling pathway may allow macrophages to calibrate their activation and function to metabolic status and nutrient availability (Fig. 6a). In contrast, this regulatory circuitry is disrupted by constitutive or aberrant activation of mTORC1, as occurs during nutrient excess or in our genetic model. As a consequence, induction of M2 polarization by the synergistic interactions of the Akt and JAK/STAT pathways is impaired (Fig. 6a,b). Conversely, elevated mTORC1 activity and consequent downregulation of Akt signalling may facilitate increased responses to LPS stimulation (Fig. 6b). Finally, we note that such 'hard-wiring' of mTORC1-Akt signalling to cytokine signalling could have relevance to other immunological contexts, given that many cytokines that regulate immune cell function engage the Akt pathway (and presumably also mTORC1).

In conclusion, our study highlights a key role of the mTORC1 pathway in control of macrophage polarization. Such control is likely to be of particular relevance for adipose tissue macrophages (ATMs). In the lean state, ATMs with a M2 phenotype maintain an anti-inflammatory environment and adipocyte insulin sensitivity, whereas in obesity, ATMs with a M1 phenotype produce inflammatory cytokines and promote insulin resistance and metabolic dysfunction. We propose that nutrient sensing by 
Acute activation of mTORC1

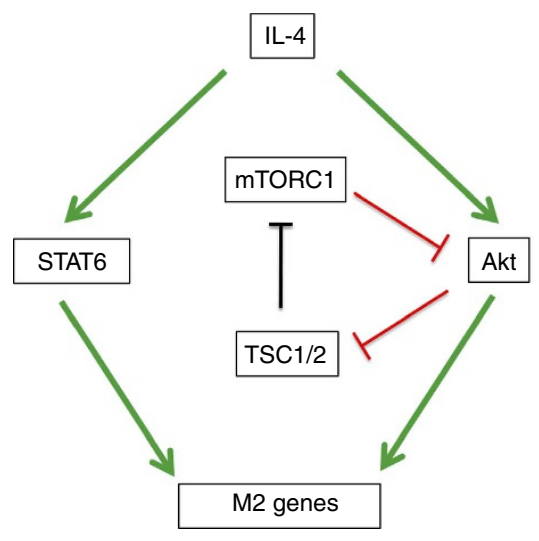

TSC deletion or chronic activation of mTORC1

b
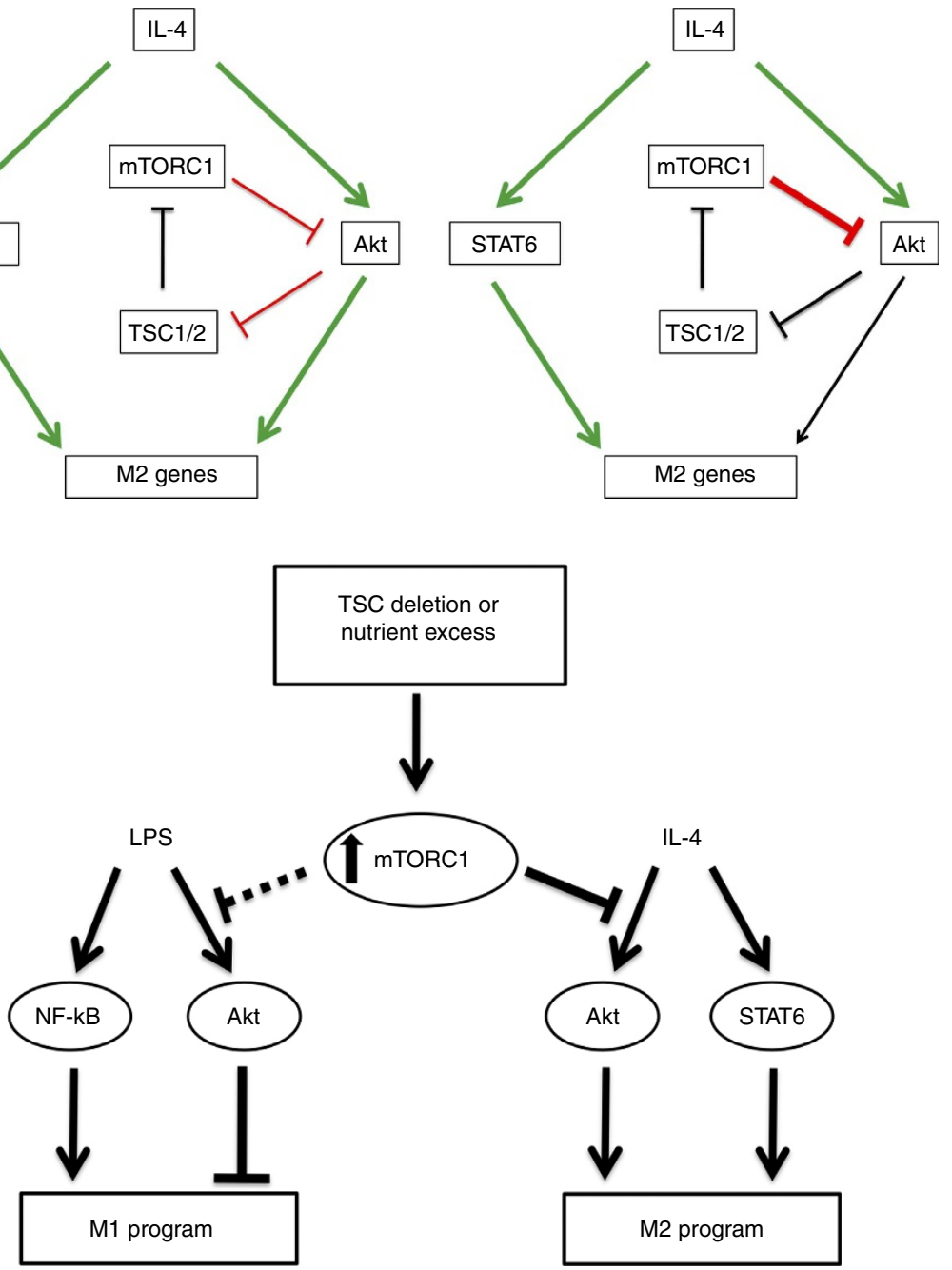

Figure 6 | Proposed model for how mTORC1 activity controls macrophage polarization. (a) Physiological induction of the Akt-mTORC1 signalling loop by IL-4 stimulation (left) allows for transient, inducible activation of the pathway, and enables Akt to synergize with the JAK/STAT pathway for M2 polarization. mTORC1 activity is also regulated by nutrient availability (not shown here), so such wiring of the signalling pathway may allow calibration of M2 activation to metabolic status (left). In contrast, constitutive or aberrant mTORC1 activation corrupts this signalling pathway and modulation of macrophage activation by metabolic inputs (right). (Green = activation; red=inhibition; black=attenuated) (b) Constitutive or aberrant activation of mTORC1 impairs the ability of macrophages to respond appropriately to polarizing stimuli. A critical mediator of this process is Akt, whose activity is downregulated by increased mTORC1 activity.

mTORC1 may directly regulate the pathophysiological switch of ATMs during obesity, extending the current model for regulation of the pathophysiological M2 to M1 switch ${ }^{62}$. Indeed, given that macrophages are critical orchestrators of diverse physiological responses, regulation of macrophage activation by the mTOR pathway may have profound consequences in many settings, including helminth infection, inflammation, allergy and tissue repair.

\section{Methods}

BMDMs. Briefly, femurs were removed from mice after CO2 euthanasia, and cells were liberated using a mortar and pestle. For macrophage differentiation, bone marrow-derived cells were plated in Petri dishes with 1640 RPMI media (10\% FCS, penicillin/streptomycin, $2 \mathrm{mM}$ L-Glutamine) supplemented with MCSF-containing L929 cell supernatant for 7 days. MCSF-differentiated macrophages were harvested and plated in tissue culture dishes for subsequent experiments. For M1-like activation, 0.5-0.7 $\times 10^{6}$ BMDMs were plated in 12-well tissue culture dishes and treated with $10 \mathrm{ng} \mathrm{ml}^{-1}$ LPS (Invivogen). For M2(a) polarization, cells were treated with $10 \mathrm{ng} \mathrm{ml}^{-1} \mathrm{IL}-4$ (Peprotech). For M2(b) polarization, cells were treated with $10 \mathrm{ng} \mathrm{ml}^{-1} \mathrm{IL}-10$ (Peprotech). For M2b polarization, cells were treated simultaneously with $10 \mathrm{ng} \mathrm{ml}^{-1}$ LPS (Invivogen) and either unopsonized sheep red blood cells (SRBC) (Lampire biological laboratories) or SRBCs opsonized with 1:400 antiSRBC immunoglobulin G (cat\# 55806 MP Biomedicals).

Mice. To generate mice with targeted deletion of $T s c 1$ in myeloid lineage cells, mice with flanking loxP Tsc1 alleles (Tsc1 ${ }^{f l f l}$ ) were crossed to LysozymeM Cre transgenic mice, both on a B6 background ${ }^{38,63}$. Cre-recombinase activity results in deletion of exons 17 and 18 of Tsc1, generating a null allele ${ }^{64}$. Male mice aged 12 weeks were used for in vivo chitin administration and male mice aged 6-8 weeks were used for in vivo IL-4 administration. For Rictor deletion in vivo, tamoxifen (VWR) was suspended in sunflower seed oil (VWR) at $10 \mathrm{mg} \mathrm{ml}^{-1}$, and $200 \mu \mathrm{l} \mathrm{per}$ $25 \mathrm{~g}$ body weight was injected into 10 -week old Rictor ${ }^{\text {fl/fl }}$ and Rictor ${ }^{f l / f l}$ UbiquitinCCreERT2 mice once daily for 7 days ${ }^{65}$. Bone marrow from such mice was used to generate Rictor-deficient macrophages. Mice were maintained at Harvard Medical School and Massachusetts Institute of Technology, and all procedures were 
performed in accordance with the guidelines set forth by the Institutional Animal Care and Use Committees at each institution.

Immunoblotting. For protein sample preparation, cells were washed twice with cold phosphate-buffered saline (PBS) following stimulation and lysed in 1\% NP-40 buffer with EDTA-free protease inhibitor tablets (Roche Diagnostics) and phosphatase inhibitors. Protein concentration in lysates was determined using the Bradford method. Equal amounts of protein were loaded onto SDS-PAGE gels and subsequently transferred to polyvinylidene difluoride membranes for immunoblotting with primary antibodies as indicated. Full blots of all immunoblots shown in the main article are included in Supplementary Fig. S9.

Antibodies and reagents. Primary antibodies were purchased from Cell Signaling (all at 1:1,000 dilution in 5\% bovine serum albumin (BSA)), except for the following: $\alpha$ - Tubulin (Sigma, 1:5,000), $\beta$-Actin (Sigma, 1:2,000), Flag-M2 (Sigma, 1:1,000) and PPAR $\gamma$ (Santa Cruz E-8, 1:1,000) and PPAR $\delta$ (Santa Cruz, 1:250). For flow cytometry, antibodies used were CD11b-PE (BD Biosciences) and F4/80-FITC (BioLegend). Inhibitors were used as follows: MK-2206 $1 \mu \mathrm{M}$ (Selleck), Akt VIII $10 \mu \mathrm{M}$ (Enzo), rapamycin $20 \mathrm{nM}$ (LC Laboratories), Troglitazone $1 \mu \mathrm{M}$ (Cayman) and GW501516 $100 \mu \mathrm{M}$ (Enzo).

Arginase assays. Arginase assay was described previously ${ }^{66}$. Briefly, $0.5 \times 10^{6}$ cells per well in 12-well plates were stimulated with IL-4 for 12-48 h. Cells were lysed in $0.1 \%$ TritonX-100 lysis buffer with protease inhibitors. Lysates with equal amounts of protein were incubated with $500 \mathrm{mM} \mathrm{L}$-Arginine for $45 \mathrm{~min}$ at $37^{\circ} \mathrm{C}$, followed by acid stop solution. The degradation of $\mathrm{L}$-arginine to urea was measured by adding $9 \%$ isonitrosopropiophenone in $100 \%$ ethanol. Absorbance was read at $540 \mathrm{~nm}$ in a microplate reader. All samples were read in triplicate.

Fatty acid oxidation. BMDMs were plated $0.7 \times 10^{6}$ cells per well in 12 -well tissue culture dishes in complete RPMI and stimulated with IL-4 for $36 \mathrm{~h}$. After stimulation, cells were washed with PBS and loaded with low-glucose Dulbecco's

Modified Eagle Medium $+2 \%$ fatty acid-free BSA (Lampire Biologicals) for 30 min at $37^{\circ} \mathrm{C}$. After $30 \mathrm{~min}$, cells were washed with PBS and given ${ }^{3} \mathrm{H}$-labelled palmitic acid ( $2 \mu \mathrm{Ci}$ per well, MP Biomedicals) in low-glucose Dulbecco's Modified Eagle Medium, with $2 \%$ fatty acid-free BSA and $0.2 \mathrm{mM}$ unlabelled oleic acid (Sigma). After $4 \mathrm{~h}, 100 \mu \mathrm{l}$ of media was collected and the isolation of ${ }^{3} \mathrm{H} 2 \mathrm{O}$ was performed using trichloroacetic acid followed by chloroform-methanol extraction. The watersoluble fraction was collected in $5 \mathrm{ml}$ of EcoLume (MP Biomedicals) scintillation fluid and counted for 5 min using a Beckman LS6500 scintillation counter. Cells were lysed in $500 \mu \mathrm{l}$ of $0.1 \mathrm{~N} \mathrm{NaOH}$ and total protein was determined using the Bradford method. Background ${ }^{3} \mathrm{H}$ was subtracted from the c.p.m. (counts per minute) value and all samples were normalized to a milligram of total protein. All samples were performed in duplicate.

Myr-Akt-transduction. To make retrovirus particles, 293T cells were co-transfected with pBabe empty vector Puro or pBabe Puro Myr Flag Human-Akt1 purchased from Addgene (plasmid 15294) along with pCL-Eco (Imgenex \#10045P) using Lipofectamine 2000 (Invitrogen). Transfected 293T media was changed the next day and placed at $32{ }^{\circ} \mathrm{C}$. Viral supernatant was collected on day 2 and day 3 post transfection. Fresh bone marrow was plated on the same day as transfection above and transduced with media containing viral supernatant (50\% viral supernatant containing pBabe empty vector or pBabe Puro Myr Flag Human-Akt1, 40\% RPMI complete media, and 10\% CMG media) on day 2 and day 3 and selected using $4 \mu \mathrm{g} \mathrm{ml}^{-1}$ puromycin on days $4-7$.

ELISA. Cytokine concentration was determined using for IL-10, TNF $\alpha$ and IL- 6 using ELISA kits purchased from BioLegend. Briefly, experimental supernatants were collected and centrifuged at 3,000 g per $5 \mathrm{~min}$. Supernatants were analysed in duplicate per manufacturers protocol.

Chromatin immunoprecipitation. For STAT6 ChIP, BMDMS $\left(5 \times 10^{6}\right)$ were plated in $6 \mathrm{~cm}$ tissue culture plates and stimulated for $2 \mathrm{~h}$ with $10 \mathrm{ng} \mathrm{ml}^{-1} \mathrm{IL}-4$ Cells were subsequently fixed with $1 \%$ formaldehyde for $10 \mathrm{~min}$ at room temperature. Formaldehyde was quenched with glycine. After collecting cells, lysis was performed using $500 \mu \mathrm{l}$ of SDS buffer (1\% SDS, $10 \mathrm{mM}$ EDTA, $50 \mathrm{mM}$ Tris-Cl pH 8) plus protease and phosphatase inhibitors. Cells were subsequently sonicated for 3 min on ice with 1-s pulses using a Misonix 4000 sonicator to shear chromatin. Following sonication, samples were diluted to $3 \mathrm{ml}$ with ChIP dilution buffer (0.01\% SDS, $1.1 \%$ Triton X-100, $1.2 \mathrm{mM}$ EDTA, $16.7 \mathrm{mM}$ Tris-Cl pH 8, $167 \mathrm{mM}$ $\mathrm{NaCl}$ ) and precleared for $1 \mathrm{~h}$ at $4{ }^{\circ} \mathrm{C}$ with protein A salmon sperm/agarose beads (Millipore). Precleared chromatin was split into $400 \mu \mathrm{l}$ aliquots for IP with either $5 \mu \mathrm{g}$ of STAT6 (M-20 ChIP grade, Santa Cruz) or for no antibody control overnight at $4^{\circ} \mathrm{C}$. IPs were incubated with protein A salmon sperm/agarose beads (Millipore) the following day for $3 \mathrm{~h}$ at $4{ }^{\circ} \mathrm{C}$. After $3 \mathrm{~h}$, beads were spun down and $\sim 10 \%$ of chromatin was taken for input and processed in parallel to IP samples. Antibody bead complexes were then washed with low salt $(0.1 \%$ SDS, $1 \%$ Triton X-100,
2 mM EDTA, $20 \mathrm{mM}$ Tris-Cl pH 8, $150 \mathrm{mM} \mathrm{NaCl})$, high salt (0.1\% SDS, $1 \%$ Triton $\mathrm{X}-100,2 \mathrm{mM}$ EDTA, $20 \mathrm{mM}$ Tris-Cl pH 8, $500 \mathrm{mM} \mathrm{NaCl}$ ) and lithium chloride $(0.25 \mathrm{M} \mathrm{LiCl}, 1 \%$ deoxycholic acid, $1 \% \mathrm{NP}-40,1 \mathrm{mM}$ EDTA, $10 \mathrm{mM}$ Tris-Cl pH 8$)$ buffers followed by two washes with TE buffer. Chromatin-antibody complexes were eluted with elution buffer ( $1 \%$ SDS $\pm 0.1 \mathrm{M}$ sodium bicarbonate) and crosslinks were reversed using sodium chloride and incubation at $60^{\circ} \mathrm{C}$ for $4 \mathrm{~h}$. Samples were then incubated $1 \mathrm{~h}$ with proteinase $\mathrm{K}$ (Roche) at $60^{\circ} \mathrm{C}$. DNA was purified using PCR purification columns (Qiagen) and used for quantitative PCR with primers generated to the Arg1 promoter region. Fold enrichment was calculated as ChIP signals divided by no antibody control and normalized to input.

Quantitative PCR. To measure gene expression in BMDMs and PECs, RNA was isolated using RNA Bee (Tel-Test) per manufacturers protocol and used to make cDNA using High Capacity cDNA Reverse Transcription Kit (Applied Biosystems). A Bio-Rad C1000 Thermocycler was used to analyse the samples under the following conditions: $95^{\circ} \mathrm{C}(5 \mathrm{~min}), 50$ cycles of $95^{\circ} \mathrm{C}(10 \mathrm{~s}), 60^{\circ} \mathrm{C}(10 \mathrm{~s})$, and $72^{\circ} \mathrm{C}(20 \mathrm{~s})$. Reaction mixture consisted of $4 \mu \mathrm{l}$ cDNA, $1.5 \mu \mathrm{l} 3 \mu \mathrm{M}$ primers for each gene used in the study $(\mathrm{F}+\mathrm{R}), 2 \mu \mathrm{l} \mathrm{H} 2 \mathrm{O}$, and $7.5 \mu \mathrm{l} 2 \times$ SYBR green (Bio-Rad). Samples from BMDMs were normalized to hypoxanthine phosphoribosyltransferase and samples from PECs were normalized to the macrophage marker CD68. Data were analysed by means of the CFX Manger Software (Bio-Rad) using the delta/delta CT method. Sequences for all qPCR primers are shown in Supplementary Table S1.

Chitin administration. Chitin (Sigma) was prepared as previously described ${ }^{48}$ Briefly, chitin was washed three times with PBS and then sonicated (Misonix Sonicator 4000) for $30 \mathrm{~min}$ on ice. The dissolved chitin was filtered and diluted with PBS to $4 \mu \mathrm{g} \mathrm{ml}^{-1} .800 \mathrm{ng}$ chitin dissolved in $200 \mu \mathrm{l}$ of PBS was injected IP and PECs were collected $48 \mathrm{~h}$ post injection.

IL-4 complex administration. Long acting IL-4 complex (IL-4c) was prepared as previously described ${ }^{47}$. Briefly, IL-4 (Peprotech) was suspended at a concentration of $500 \mu \mathrm{g} \mathrm{ml}^{-1}$ and mixed with anti-mouse IL-4 (BioXcell clone 11b11) at a molar ratio of 2:1 (weight 1:5) and incubated 1-2 min at room temperature. IL-4c was suspended in normal saline to a concentration of $25 \mu \mathrm{g} \mathrm{ml}{ }^{-1}$ IL-4 and $125 \mu \mathrm{g} \mathrm{ml}^{-1}$ of $11 \mathrm{~b} 11$. Each mouse was injected IP with $200 \mu \mathrm{l}$ of IL-4c (5 $\mu \mathrm{g}$ IL-4 and $25 \mu \mathrm{g} 11 \mathrm{~b} 11)$ on day 0 and 2 , and PECs were collected on day 4 .

Dual luciferase assays. BMDMs were electroporated using Amaxa nucleofector and mouse macrophage nucleofector kit (Lonza) with PPAR-Firefly luciferase plasmid (C.H. Lee, Harvard School of Public Health) or STAT6-Firefly luciferase (purchased from Addgene-plasmid \#35554) along with Renilla-Luciferase plasmid as a transfection control. The PPAR- Firefly luciferase plasmid consists of three copies of the Acox1 PPAR response element ${ }^{67}$ upstream of an SV40 minimal promoter. BMDMs were treated with IL-4 and/or troglitazone $4 \mathrm{~h}$ post electroporation for $24 \mathrm{~h}$. Cell lysates were collected and analysed using the Promega Dual-Luciferase Reporter Assay System.

Statistical analysis. Statistical analysis was carried out using Prism (GraphPad) software. The student's $t$-test was used to determine statistical significance, defined as $P<0.05$.

\section{References}

1. Sica, A. \& Mantovani, A. Macrophage plasticity and polarization: in vivo veritas. J. Clin. Invest. 122, 787-795 (2012).

2. Gordon, S. \& Martinez, F. O. Alternative activation of macrophages: mechanism and functions. Immunity 32, 593-604 (2010).

3. Chawla, A. Control of macrophage activation and function by PPARs. Circ. Res 106, 1559-1569 (2010)

4. Odegaard, J. I. et al. Macrophage-specific PPARgamma controls alternative activation and improves insulin resistance. Nature 447, 1116-1120 (2007).

5. Kang, K. et al. Adipocyte-derived Th2 cytokines and myeloid PPARdelta regulate macrophage polarization and insulin sensitivity. Cell Metab. 7, 485-495 (2008).

6. Van den Bossche, J. et al. Pivotal advance: arginase-1-independent polyamine production stimulates the expression of IL-4-induced alternatively activated macrophage markers while inhibiting LPS-induced expression of inflammatory genes. J. Leukoc. Biol. 91, 685-699 (2012).

7. Murray, P. J. \& Wynn, T. A. Protective and pathogenic functions of macrophage subsets. Nat. Rev. Immunol. 11, 723-737 (2011).

8. Cramer, T. et al. HIF-1alpha is essential for myeloid cell-mediated inflammation. Cell 112, 645-657 (2003).

9. Vats, D. et al. Oxidative metabolism and PGC-1beta attenuate macrophagemediated inflammation. Cell Metab. 4, 13-24 (2006).

10. Howell, J. J. \& Manning, B. D. mTOR couples cellular nutrient sensing to organismal metabolic homeostasis. Trends Endocrinol. Metab. 22, 94-102 (2011). 
11. Duvel, K. et al. Activation of a metabolic gene regulatory network downstream of mTOR complex 1. Mol. Cell 39, 171-183 (2010).

12. Tee, A. R., Fingar, D. C., Manning, B. D., Kwiatkowski, D. J., Cantley, L. C. \& Blenis, J. Tuberous sclerosis complex-1 and -2 gene products function together to inhibit mammalian target of rapamycin (mTOR)-mediated downstream signaling. Proc. Natl Acad. Sci. USA 99, 13571-13576 (2002).

13. Huang, J. \& Manning, B. D. A complex interplay between Akt, TSC2 and the two mTOR complexes. Biochem. Soc. Trans 37, 217-222 (2009).

14. Chi, H. Regulation and function of mTOR signalling in T cell fate decisions. Nat. Rev. Immunol. 12, 325-338 (2012).

15. Sengupta, S., Peterson, T. R. \& Sabatini, D. M. Regulation of the mTOR complex 1 pathway by nutrients, growth factors, and stress. Mol. Cell 40, 310-322 (2010)

16. Chong-Kopera, H. et al. TSC1 stabilizes TSC2 by inhibiting the interaction between TSC2 and the HERC1 ubiquitin ligase. J. Biol. Chem. 281, 8313-8316 (2006).

17. Pan, H., O’Brien, T. F., Zhang, P. \& Zhong, X. P. The role of tuberous sclerosis complex 1 in regulating innate immunity. J. Immunol. 188, 3658-3666 (2012).

18. Fingar, D. C., Salama, S., Tsou, C., Harlow, E. \& Blenis, J. Mammalian cell size is controlled by mTOR and its downstream targets S6K1 and 4EBP1/eIF4E. Genes Dev. 16, 1472-1487 (2002).

19. Mantovani, A., Sica, A., Sozzani, S., Allavena, P., Vecchi, A. \& Locati, M. The chemokine system in diverse forms of macrophage activation and polarization. Trends Immunol. 25, 677-686 (2004).

20. Filardy, A. A. et al. Proinflammatory clearance of apoptotic neutrophils induces an IL-12(low)IL-10(high) regulatory phenotype in macrophages. J. Immunol. 185, 2044-2050 (2010).

21. Martinez, F. O., Sica, A., Mantovani, A. \& Locati, M. Macrophage activation and polarization. Front. Biosci. 13, 453-461 (2008).

22. Szanto, A. et al. STAT6 transcription factor is a facilitator of the nuclear receptor PPARgamma-regulated gene expression in macrophages and dendritic cells. Immunity 33, 699-712 (2010)

23. Acacia de Sa Pinheiro, A. et al. IL-4 induces a wide-spectrum intracellular signaling cascade in CD8 + T cells. J. Leukoc. Biol. 81, 1102-1110 (2007).

24. Wang, I. M., Lin, H., Goldman, S. J. \& Kobayashi, M. STAT-1 is activated by IL-4 and IL-13 in multiple cell types. Mol. Immunol. 41, 873-884 (2004).

25. Bhattacharjee, A. et al. IL-4 and IL-13 employ discrete signaling pathways for target gene expression in alternatively activated monocytes/macrophages. Free Radic. Biol. Med. 54, 1-16 (2013).

26. Tontonoz, P. et al. Adipocyte-specific transcription factor ARF6 is a heterodimeric complex of two nuclear hormone receptors, PPAR gamma and RXR alpha. Nucleic. Acids. Res. 22, 5628-5634 (1994).

27. Tontonoz, P., Nagy, L., Alvarez, J. G., Thomazy, V. A. \& Evans, R. M. PPARgamma promotes monocyte/macrophage differentiation and uptake of oxidized LDL. Cell 93, 241-252 (1998).

28. Reilly, S. M. \& Lee, C. H. PPAR delta as a therapeutic target in metabolic disease. FEBS Lett. 582, 26-31 (2008)

29. Wurster, A. L., Withers, D. J., Uchida, T., White, M. F. \& Grusby, M. J. Stat6 and IRS-2 cooperate in interleukin 4 (IL-4)-induced proliferation and differentiation but are dispensable for IL-4-dependent rescue from apoptosis. Mol. Cell Biol. 22, 117-126 (2002).

30. Heller, N. M. et al. Type I IL-4Rs selectively activate IRS-2 to induce target gene expression in macrophages. Sci. Signal 1, ra17 (2008).

31. Manning, B. D. \& Cantley, L. C. AKT/PKB signaling: navigating downstream. Cell 129, 1261-1274 (2007).

32. Fang, X. et al. Convergence of multiple signaling cascades at glycogen synthase kinase 3: edg receptor-mediated phosphorylation and inactivation by lysophosphatidic acid through a protein kinase C-dependent intracellular pathway. Mol. Cell Biol. 22, 2099-2110 (2002).

33. Cross, D. A., Alessi, D. R., Cohen, P., Andjelkovich, M. \& Hemmings, B. A. Inhibition of glycogen synthase kinase- 3 by insulin mediated by protein kinase B. Nature 378, 785-789 (1995).

34. Eldar-Finkelman, H., Seger, R., Vandenheede, J. R. \& Krebs, E. G. Inactivation of glycogen synthase kinase- 3 by epidermal growth factor is mediated by mitogen-activated protein kinase/p90 ribosomal protein S6 kinase signaling pathway in NIH/3T3 cells. J. Biol. Chem. 270, 987-990 (1995).

35. Zhang, H. H., Lipovsky, A. I., Dibble, C. C., Sahin, M. \& Manning, B. D. S6K1 regulates GSK3 under conditions of mTOR-dependent feedback inhibition of Akt. Mol. Cell 24, 185-197 (2006).

36. Harrington, L. S. et al. The TSC1-2 tumor suppressor controls insulinPI3K signaling via regulation of IRS proteins. J. Cell Biol. 166, 213-223 (2004)

37. Shah, O. J., Wang, Z. \& Hunter, T. Inappropriate activation of the TSC/Rheb/ mTOR/S6K cassette induces IRS1/2 depletion, insulin resistance, and cell survival deficiencies. Curr. Biol. 14, 1650-1656 (2004)
38. Yecies, J. L. et al. Akt stimulates hepatic SREBP1c and lipogenesis through parallel mTORC1-dependent and independent pathways. Cell Metab. 14, 21-32 (2011).

39. Fritsche, L. et al. Insulin-induced serine phosphorylation of IRS-2 via ERK1/2 and mTOR: studies on the function of Ser675 and Ser907. Am. J. Physiol. Endocrinol. Metab. 300, E824-E836 (2011).

40. Harrington, L. S., Findlay, G. M. \& Lamb, R. F. Restraining PI3K: mTOR signalling goes back to the membrane. Trends. Biochem. Sci. 30, 35-42 (2005).

41. Yu, Y. et al. Phosphoproteomic analysis identifies Grb10 as an mTORC1 substrate that negatively regulates insulin signaling. Science 332, 1322-1326 (2011).

42. Hsu, P. P. et al. The mTOR-regulated phosphoproteome reveals a mechanism of mTORC1-mediated inhibition of growth factor signaling. Science 332, 1317-1322 (2011).

43. Holt, L. J. \& Siddle, K. Grb10 and Grb14: enigmatic regulators of insulin action--and more? Biochem. J. 388, 393-406 (2005).

44. Wick, K. R. et al. Grb10 inhibits insulin-stimulated insulin receptor substrate (IRS)-phosphatidylinositol 3-kinase/Akt signaling pathway by disrupting the association of IRS-1/IRS-2 with the insulin receptor. J. Biol. Chem. 278, 8460-8467 (2003).

45. Vecchione, A., Marchese, A., Henry, P., Rotin, D. \& Morrione, A. The Grb10/Nedd4 complex regulates ligand-induced ubiquitination and stability of the insulin-like growth factor I receptor. Mol. Cell Biol. 23, 3363-3372 (2003).

46. Mora, A., Komander, D., van Aalten, D. M. \& Alessi, D. R. PDK1, the master regulator of AGC kinase signal transduction. Semin. Cell Dev. Biol. 15, 161-170 (2004).

47. Jenkins, S. J. et al. Local macrophage proliferation, rather than recruitment from the blood, is a signature of TH2 inflammation. Science 332, 1284-1288 (2011).

48. Satoh, T. et al. The Jmjd3-Irf4 axis regulates M2 macrophage polarization and host responses against helminth infection. Nat. Immunol. 11, 936-944 (2010).

49. Reese, T. A. et al. Chitin induces accumulation in tissue of innate immune cells associated with allergy. Nature 447, 92-96 (2007).

50. Weichhart, T. et al. The TSC-mTOR signaling pathway regulates the innate inflammatory response. Immunity 29, 565-577 (2008).

51. Chen, W. et al. Macrophage-induced tumor angiogenesis is regulated by the TSC2-mTOR pathway. Cancer Res. 72, 1363-1372 (2012).

52. O'Neill, L. A. \& Hardie, D. G. Metabolism of inflammation limited by AMPK and pseudo-starvation. Nature 493, 346-355 (2013).

53. Biswas, S. K. \& Mantovani, A. Macrophage plasticity and interaction with lymphocyte subsets: cancer as a paradigm. Nat. Immunol. 11, 889-896 (2010).

54. Wills-Karp, M. \& Finkelman, F. D. Untangling the complex web of IL-4- and IL-13-mediated signaling pathways. Sci. Signal. 1, pe55 (2008).

55. Song, M. S., Salmena, L. \& Pandolfi, P. P. The functions and regulation of the PTEN tumour suppressor. Nat. Rev. Mol. Cell Biol. 13, 283-296 (2012).

56. Luyendyk, J. P. et al. Genetic analysis of the role of the PI3K-Akt pathway in lipopolysaccharide-induced cytokine and tissue factor gene expression in monocytes/macrophages. J. Immunol. 180, 4218-4226 (2008).

57. Fan, W. et al. FoxO1 regulates Tlr4 inflammatory pathway signalling in macrophages. EMBO J. 29, 4223-4236 (2010).

58. Arranz, A. et al. Akt1 and Akt2 protein kinases differentially contribute to macrophage polarization. Proc. Natl. Acad. Sci. USA 109, $9517-9522$ (2012).

59. Guertin, D. A. et al. Ablation in mice of the mTORC components raptor, rictor or mLST8 reveals that mTORC2 is required for signaling to Akt-FOXO and PKCalpha, but not S6K1. Dev. Cell 11, 859-871 (2006).

60. Jacinto, E. et al. SIN1/MIP1 maintains rictor-mTOR complex integrity and regulates Akt phosphorylation and substrate specificity. Cell 127, 125-137 (2006).

61. Laplante, M. \& Sabatini, D. M. mTOR signaling in growth control and disease. Cell 149, 274-293 (2012).

62. Chawla, A., Nguyen, K. D. \& Goh, Y. P. Macrophage-mediated inflammation in metabolic disease. Nat. Rev. Immunol. 11, 738-749 (2011).

63. Clausen, B. E. Conditional gene targeting in macrophages and granulocytes using LysMcre mice. Transgenic Res. 8, 265-277 (1999).

64. Kwiatkowski, D. J. et al. A mouse model of TSC1 reveals sex-dependent lethality from liver hemangiomas, and up-regulation of p70S6 kinase activity in Tsc1 null cells. Hum. Mol. Genet. 11, 525-534 (2002).

65. Lamming, D. W. et al. Rapamycin-induced insulin resistance is mediated by mTORC2 loss and uncoupled from longevity. Science 335, 1638-1643 (2012).

66. Sinha, P., Clements, V. K. \& Ostrand-Rosenberg, S. Reduction of myeloidderived suppressor cells and induction of M1 macrophages facilitate the rejection of established metastatic disease. J. Immunol. 174, 636-645 (2005).

67. Liu, S. et al. Role of peroxisome proliferator-activated receptor $\{$ delta $\} /\{$ beta $\}$ in hepatic metabolic regulation. J. Biol. Chem. 286, 1237-1247 (2011). 


\section{Acknowledgements}

This project was supported by a NIH grant R01AI102964 (to T.H.). A.J.C. is a recipient of a Ford Foundation Predoctoral Fellowship. B.D.M. was supported by a NIH grant R01-CA122617 and I.B.-S. by a LAM Foundation Fellowship. D.M.S. was funded in part by a Julie Martin Mid-Career Award in Aging Research from the American Federation of Aging Research (AFAR) and is an Investigator of the Howard Hughes Medical Institute. D.W.L. is supported by a K99/R00 award from the NIH/NIA (1K99AG041765-01A1). We also thank C.H. Lee for critical reading of the manuscript and S.H. Liu for technical advice.

\section{Author contributions}

V.B. and A.J.C. designed and performed the experiments, analysed the data and wrote the paper. T.H. supervised the project, including experimental design and data analysis, and edited the paper. I.B.-S. and D.W.L. contributed technical expertise. D.M.S. and B.D.M. provided reagents and mice.

\section{Additional information}

Supplementary Information accompanies this paper at http://www.nature.com/ naturecommunications

Competing financial interests: The authors declare no competing financial interests.

Reprints and permission information is available online at http://npg.nature.com/ reprintsandpermissions/

How to cite this article: Byles, V. et al. The TSC-mTOR pathway regulates macrophage polarization. Nat. Commun. 4:2834 doi: 10.1038/ncomms3834 (2013). 